Supporting Information

\title{
Dual Role of Zirconium Oxoclusters in Hybrid Nanoparticles: Cross- Linkers and Catalytic Sites
}

Cesare Benedetti, ${ }^{\dagger}$ Alessandro Cazzolaro, ${ }^{\S}$ Mauro Carraro, ${ }^{\S}$ Robert Graf, ${ }^{\dagger}$ Katharina Landfester, ${ }^{\dagger}$ Silvia Gross, ${ }^{*}, * \neq$ and Rafael Muñoz-Espi ${ }^{*}+, \neq \neq$

${ }^{\dagger}$ Max Planck Institute for Polymer Research, Ackermannweg 10, 55128 Mainz, Germany

${ }^{\S}$ Dipartimento di Scienze Chimiche, Università degli Studi di Padova, Via Marzolo 1, 35131 Padova, Italy

${ }^{¥}$ ICMATE-CNR, Via Marzolo 1, 35131 Padova, Italy

${ }^{\ddagger}$ Institut de Ciència dels Materials (ICMUV), Universitat de València, c/ Catedràtic José Beltrán 2, 46980 Paterna, Spain

*E-mails: silvia.gross@unipd.it (Silvia Gross); rafael.munoz@uv.es (Rafael Muñoz-Espí) 


\section{Contents}

1. Structure of Surfactants

2. Determination of Remaining Surfactant (SDS)

3. ${ }^{1} \mathrm{H}$ NMR Spectra

4. Fourier Transforms of the X-ray Absorption Fine Structure (EXAFS)

5. Solid State NMR

6. Swelling Behavior

7. Differential Scanning Calorimetry (DSC)

8. Catalytic Experiments

8.1. Kinetic traces for S10, L7, S7, L5 and S5

8.2. FTIR after catalysis

8.3. SEM images after catalysis

8.4. ICP after catalysis

8.5. Kinetic traces in the presence of $\mathbf{L 1 0}$ and $\mathbf{L} 7$ for the first 30minutes

8.6. Recycling of the catalysts 


\section{Structure of the Surfactants}

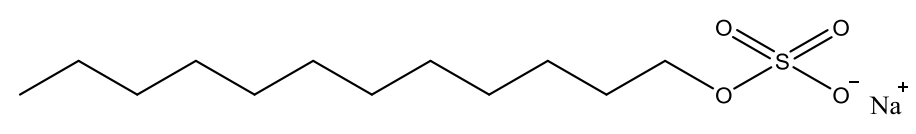

Sodium dodecyl sulfate

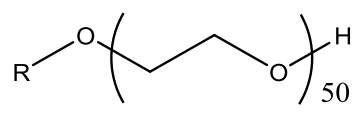

$R=$ Linear, saturated $C_{16} C_{18}$ fatty alcohol

Lutensol AT50

Scheme S1. Chemical structures of the surfactants used in the work

\section{Determination of Remaining Surfactant (SDS)}

The evaluation of SDS in solution (not adsorbed on the nanoparticles) was done by using Stains-All dye, as suggested by F. Rusconi et al. (Anal. Biochem. 2001, 295, 31-37). All particles used in the work were washed; comparisons with unwashed samples for samples S0, S5 and S10 are shown in Table S1. Taking into account the initial amount used in the miniemulsion process, the unwashed samples should contain an amount of surfactant of about $1.10 \mathrm{wt} \%$, but lower values were obtained for the samples, which indicates that part of the surfactant is entrapped in the nanoparticles and cannot be detected. An interesting outcome is the increase of the non-detectable surfactant content with the amount of $\mathbf{Z r}_{\mathbf{4}}$ in the nanoparticles matrix. This effect could be ascribed to the affinity between the surfactant and the oxocluster, and it could explain the higher concentration of SDS at the particle surface observed for sample S10 in Figure 3. Regarding the detected SDS content (or SDS in solution), we could also observe a decrease in the amount of "removed SDS" at higher amount of $\mathbf{Z r}_{\mathbf{4}}$, confirming a probable further interaction between the SDS and $\mathbf{Z} \mathbf{r}_{4}$. 
Table S1. Evaluation of SDS amount by using Stains-All dye.

\begin{tabular}{|c|c|c|c|c|}
\hline Samples & $\begin{array}{c}\text { Theoretical SDS } \\
\text { content }(\%)^{\mathrm{b}}\end{array}$ & $\begin{array}{c}\text { Detected SDS } \\
\text { content }(\%)^{\mathrm{c}}\end{array}$ & $\begin{array}{l}\text { Non-detectable SDS } \\
\text { content }(\%)^{\mathrm{d}}\end{array}$ & Removed SDS $(\%)^{\mathrm{e}}$ \\
\hline $\mathrm{S10}^{\mathrm{a}}$ & 1.10 & 0.02 & 1.08 & - \\
\hline S10 & nd & 0.02 & - & 34 \\
\hline $\mathbf{S 5}^{\mathrm{a}}$ & 1.10 & 0.13 & 0.97 & - \\
\hline S5 & nd & 0.02 & - & 82 \\
\hline $\mathrm{SO}^{\mathrm{a}}$ & 1.10 & 0.36 & 0.75 & - \\
\hline So & nd & 0.02 & - & 95 \\
\hline
\end{tabular}

[a] Before the surfactant removal. [b] Calculated taking into account the starting amounts in the miniemulsion process. [c] By absorbance measurements recorded at $438 \mathrm{~nm}$ on a Tecan Infinite M1000 plate reader. The SDS trapped in the nanoparticles cannot be detected with this method. [d] Difference between the theoretical and the detected SDS contents. [e] Ratio in \% between the sample after and before surfactant removal.

\section{3. ${ }^{1}$ H NMR Spectra}

To investigate the interaction between the $\mathrm{Zr}$ ions and the surfactants, ${ }^{1} \mathrm{H}$ NMR spectra were recorded for solutions in toluene and in THF of $\mathbf{Z r}_{4}$, Lutensol AT50, SDS, and the mixtures of the $\mathbf{Z r}_{4}$ with both surfactant solutions.

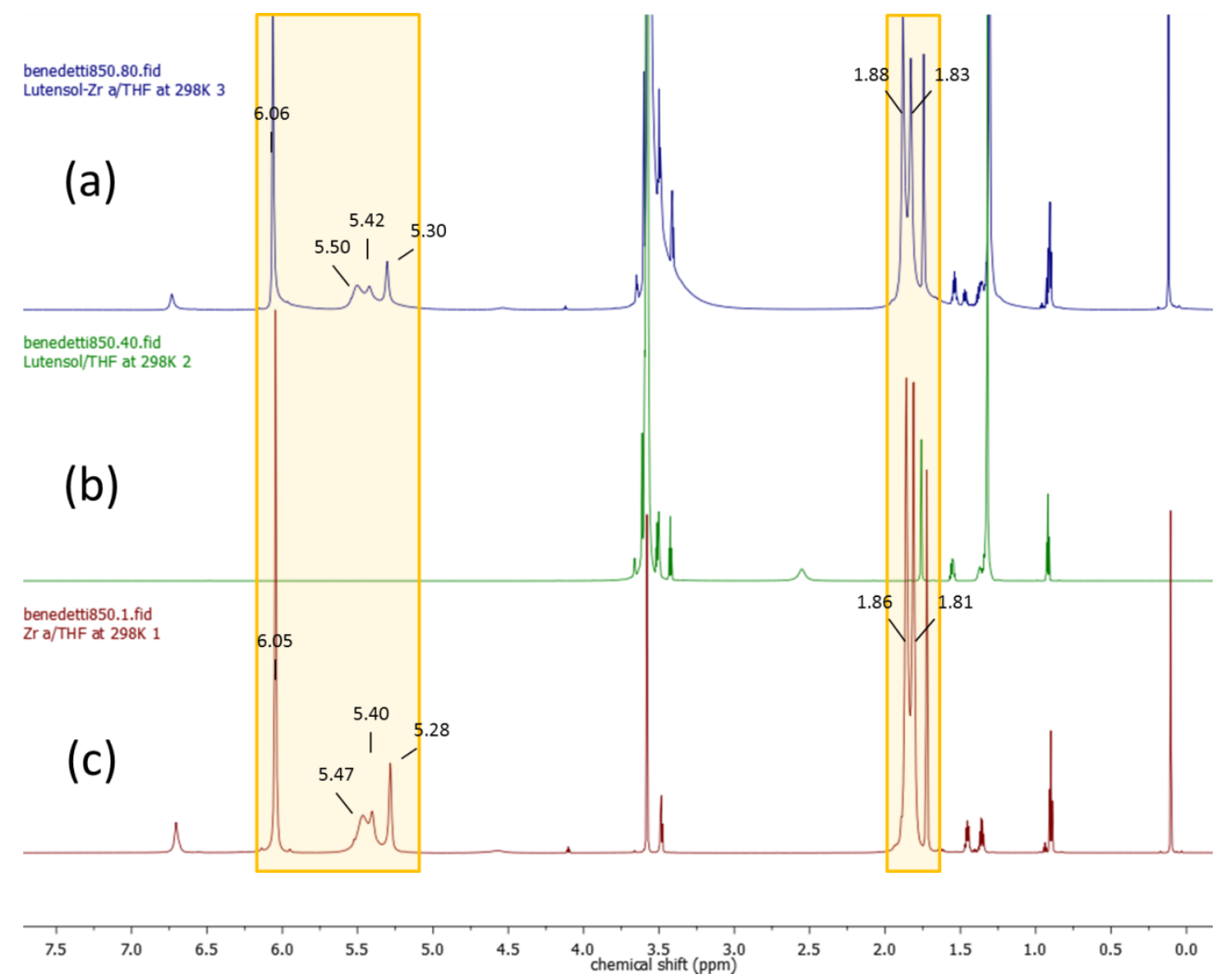

Figure S1. ${ }^{1} \mathrm{H}$ NMR spectra of (a) $\mathbf{Z r}_{4}$, (b) Lutensol AT50, and (c) a mixture of $\mathbf{Z r}_{4}$ and Lutensol AT50, all in THF. 


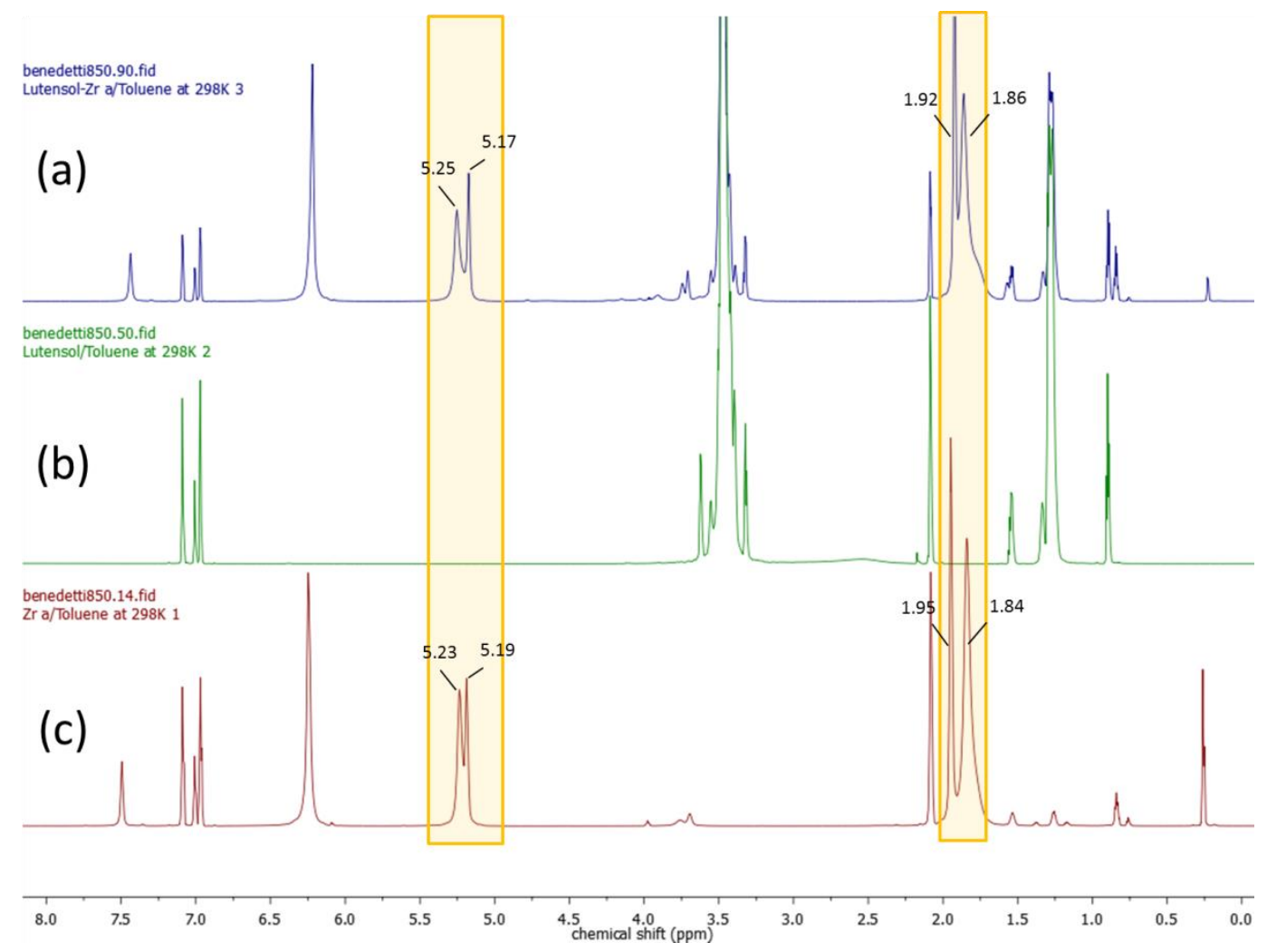

Figure S2. ${ }^{1} \mathrm{H}$ NMR spectra of (a) $\mathbf{Z} \mathbf{r}_{4}$, (b) Lutensol AT50, and (c) a mixture of $\mathbf{Z r}_{4}$ and Lutensol AT50, all in toluene.

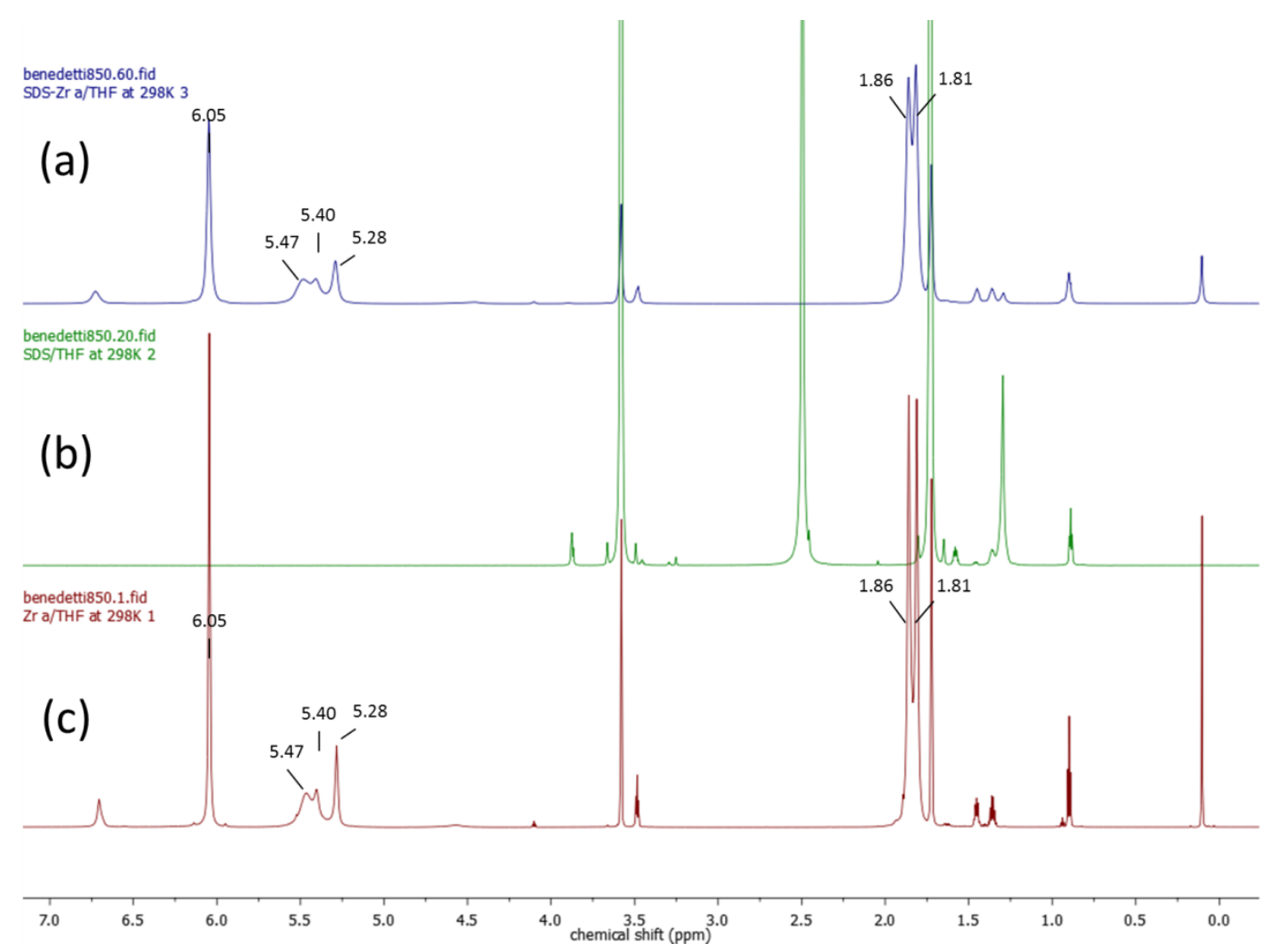

Figure S3. ${ }^{1} \mathrm{H}$ NMR spectra of (a) $\mathbf{Z} \mathbf{r}_{4}$, (b) SDS, and (c) a mixture of $\mathbf{Z r}_{4}$ and SDS, all in THF. 


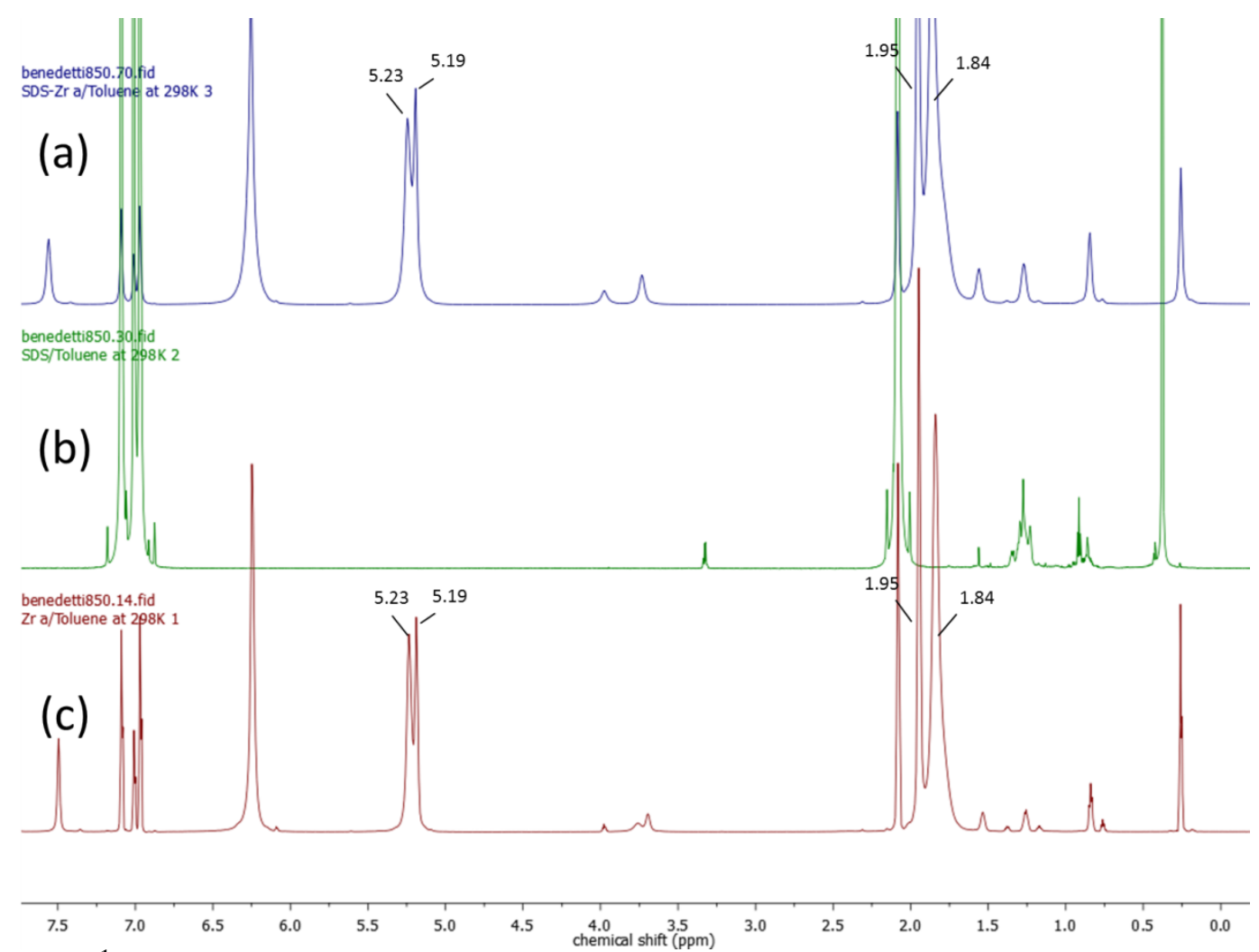

Figure S4. ${ }^{1} \mathrm{H}$ NMR spectra of (a) $\mathbf{Z} \mathbf{r}_{4}$, (b) SDS, and (c) a mixture of $\mathbf{Z r}_{4}$ and SDS, all in toluene.

\section{Fourier Transforms of the X-ray Absorption Fine Structure (EXAFS)}

(a)

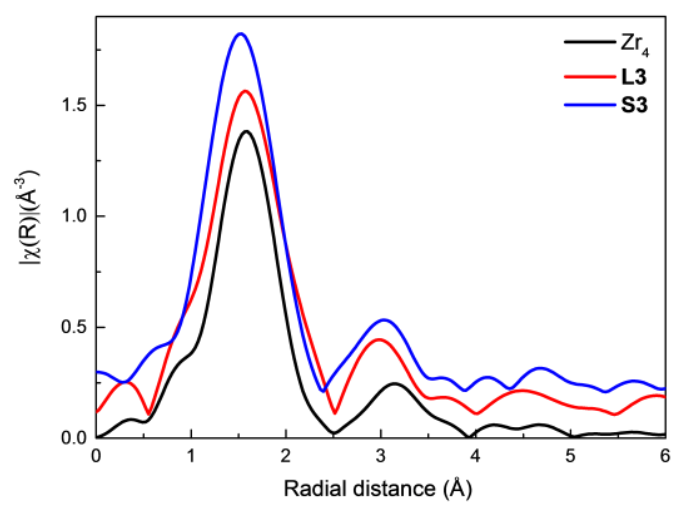

(b)

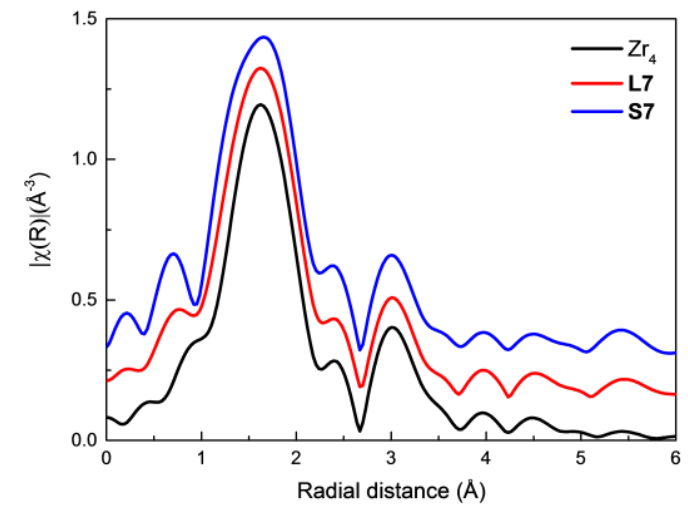

Figure S5. Fourier Transforms of the EXAFS data for $\mathbf{Z r}_{4}$ oxocluster and $\mathbf{Z r}_{\mathbf{4}}$ oxocluster covalently cross-linked into the hybrid materials nanoparticles with a weight ratio of (a) $3 \%$ and (b) $7 \%$ with respect to PMMA 


\section{Solid State NMR}

For the quantitative evaluation of the relative amount of $\mathbf{Z r}_{\mathbf{4}}$ free (unreacted) double bonds on the prepared hybrid poly(MMA-co-Z $\left.\mathbf{Z r}_{4}\right)$ nanoparticles, ${ }^{13} \mathrm{C}$ CP-MAS NMR spectra were recorded for samples S0, S3, S5, S7, and $\mathbf{Z r}_{4}$ (Figure S2). The broad signal at about $18 \mathrm{ppm}$ arises from the superposition of the intrinsically broad methyl signal of PMMA and the narrow one of $\mathbf{Z r}_{4}$. The main chain signals of PMMA appear between 40 and $60 \mathrm{ppm}$, together with that of the methoxy carbon. Two weak ${ }^{13} \mathrm{C}$ NMR signals, at 125 and $140 \mathrm{ppm}$,

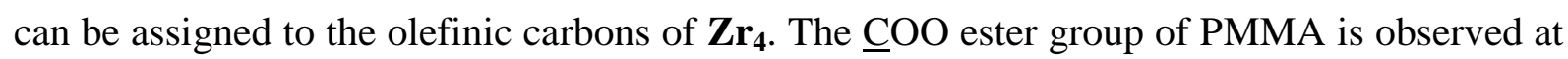
$178 \mathrm{ppm}$. The comparison NMR spectra of hybrid poly(MMA-co- $\left.\mathbf{Z r}_{\mathbf{4}}\right)$ nanoparticles with the NMR spectra of pristine $\mathbf{Z r}_{\mathbf{4}}$ and $\mathbf{S 0}$ provides information on the modifications occurring upon hybrid formation. PMMA signals in the spectra of the hybrid and of pristine polymer appear substantially identical, indicating the similarity of the main structural features of PMMA in the presence or absence of the oxoclusters. In the spectrum of the pristine oxocluster $\mathbf{Z r}_{4}$ every signal corresponding to a certain carbon nucleus appears structured in a variable number of peaks. Because of the low amount of oxoclusters embedded in the hybrid materials, their signals are largely decreased in intensity, and the spectral resolution between different sites of the oxoclusters is almost completely lost due to local heterogeneities of the hybrid materials.

Finally, the NMR signals of the free $\mathbf{Z r}_{4}$ double bonds observed at $140 \mathrm{ppm}$ were evaluated by integration and normalized to the signal of the COO ester group of PMMA at $178 \mathrm{ppm}$. The signals of $\mathbf{S 3 ,} \mathbf{S 5}$, and $\mathbf{S 7}$ are compared with the ones of the unreacted mixtures containing S0 in addition to 3, 5, and $7 \mathrm{wt} \%$ of $\mathbf{Z r}_{4}$, respectively. Since, the mixtures of S0 and $\mathbf{Z r}_{\mathbf{4}}$ have not been polymerized, the signal intensity of the $\mathbf{Z r}_{\mathbf{4}}$ double bonds are considered fully unreacted. The amount of reacted $\mathbf{Z r}_{\mathbf{4}}$ double bonds can be estimated from the ratio between signal intensities in the hybrid samples and in the mixture. 


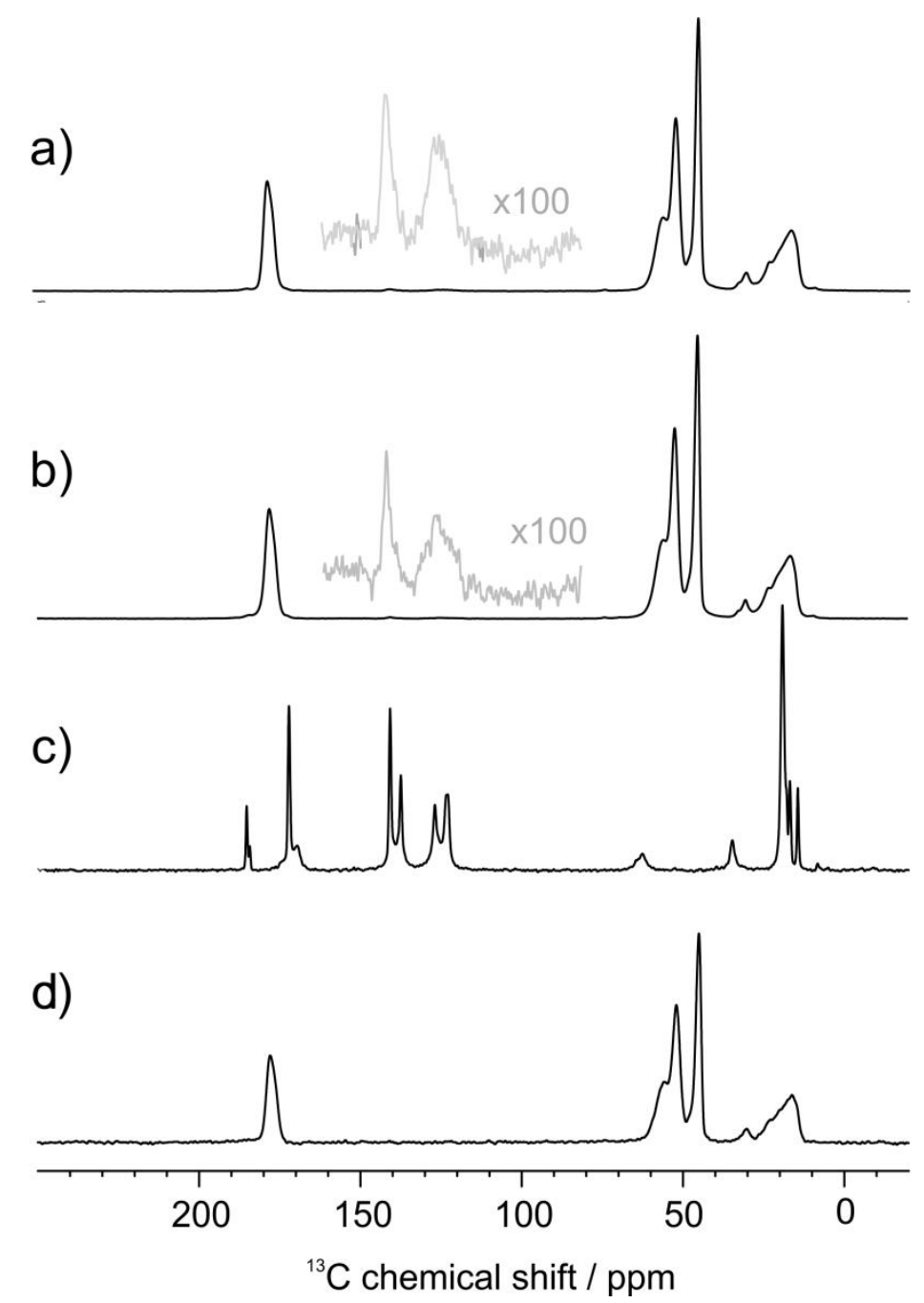

Figure S6. ${ }^{13} \mathrm{C}$ CP-MAS spectra of (a) a mixture of So (PMMA nanoparticles) and $\mathbf{Z r}_{\mathbf{4}} \mathbf{5 \%}$, (b) $\mathbf{S 5}$, (c) $\mathbf{Z r}_{4}$ oxocluster and (d) S0.

Table S2. Evaluation of the unreacted double bonds through ${ }^{13} \mathrm{C}$ CP-MAS

\begin{tabular}{cccc}
\hline Content of $\mathbf{Z r}_{\mathbf{4}}$ (wt\%) & $\begin{array}{c}\text { poly(MMA-co-Zr } \\
\text { nanoparticles }(\%)\end{array}$ & $\begin{array}{c}\text { Mixture of } \mathbf{Z r}_{4} \text { and } \mathbf{S 0} \\
\text { nanoparticles }(\%)\end{array}$ & $\begin{array}{c}\text { Unreacted } \mathbf{Z r}_{4} \text { double } \\
\text { bonds(\%) }\end{array}$ \\
\hline $\mathbf{3}$ & $0.47(\mathbf{S 3})$ & 1.05 & $45 \%$ \\
$\mathbf{5}$ & $1.04(\mathbf{S 5})$ & 3.02 & $35 \%$ \\
$\mathbf{7}$ & $0.97(\mathbf{S 7})$ & 1.60 & $60 \%$ \\
\hline
\end{tabular}




\section{Swelling behavior}

(a)

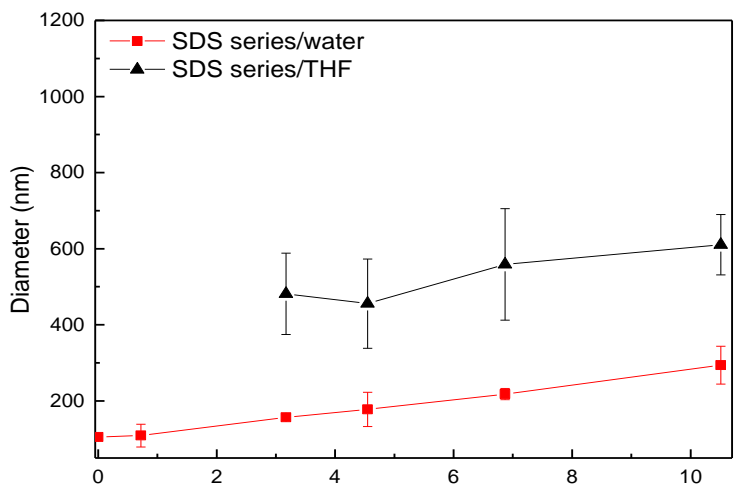

(b)

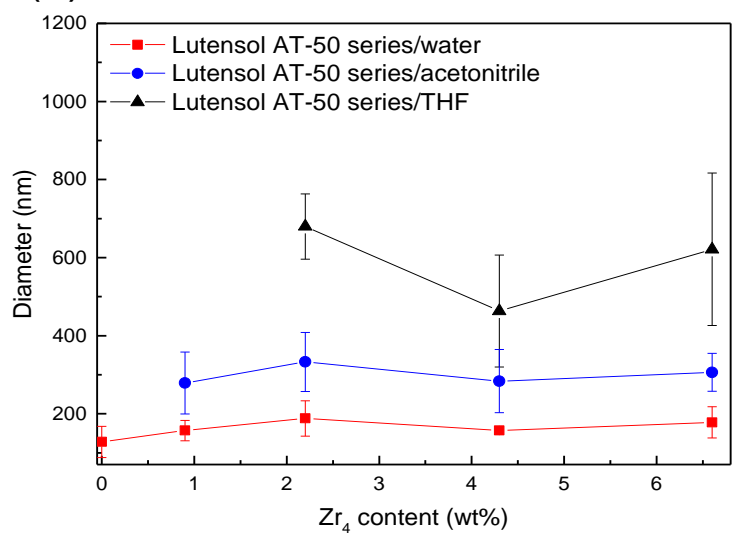

Figure S7. DLS measurement of the hydrodynamic diameter of hybrid poly(MMA-co- $\left.\mathbf{Z r}_{4}\right)$ nanoparticles (a) in water and THF for SDS samples and (b) additionally in acetonitrile for Lutensol AT50 samples as a function of $\mathrm{Zr}_{4}$ content. 


\section{Differential Scanning Calorimetry (DSC)}

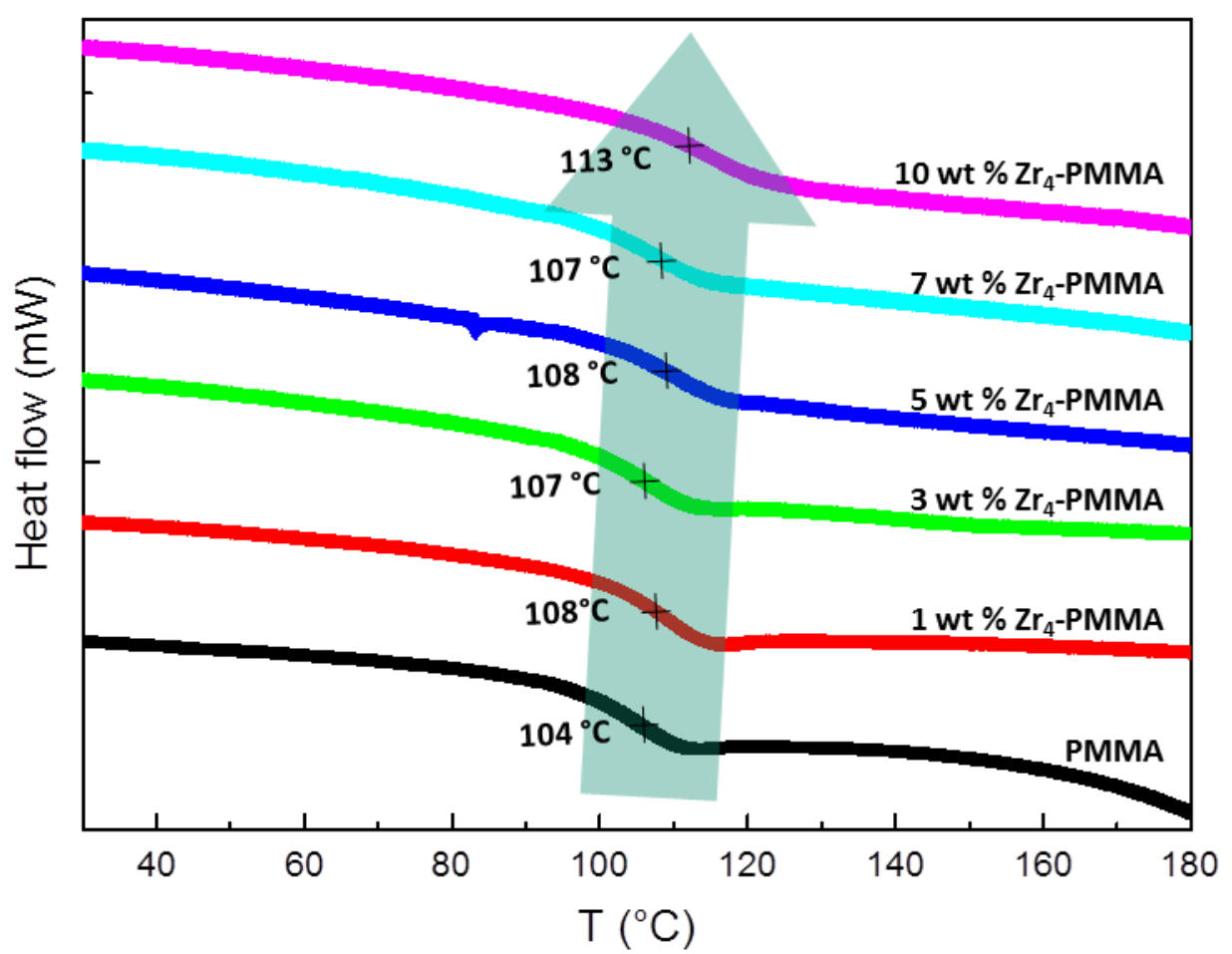

Figure S8. DSC curves of hybrid poly(MMA-co-Z $\left.\mathbf{Z r}_{\mathbf{4}}\right)$ nanoparticles prepared with SDS. The glass transition temperature of the samples shown is calculated from the first derivative. 


\section{Catalytic Experiments}

\subsection{Kinetic traces for $\mathrm{S10}, \mathrm{L7}, \mathrm{S7}, \mathrm{L5}$, and $\mathrm{S5}$}

(a)

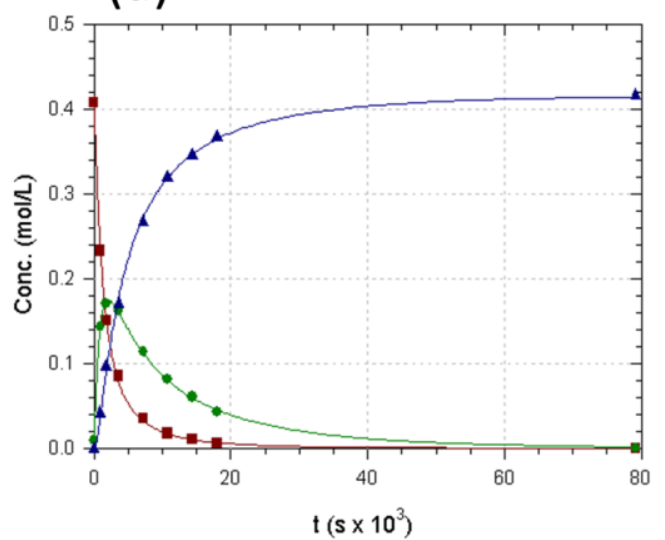

(c)

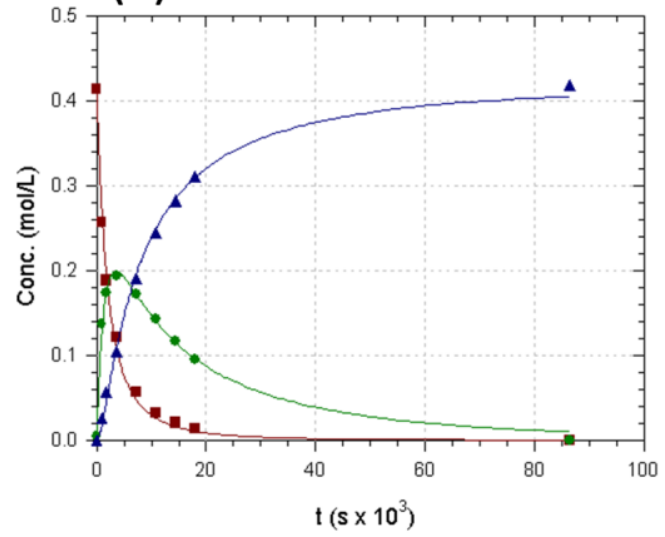

(e)

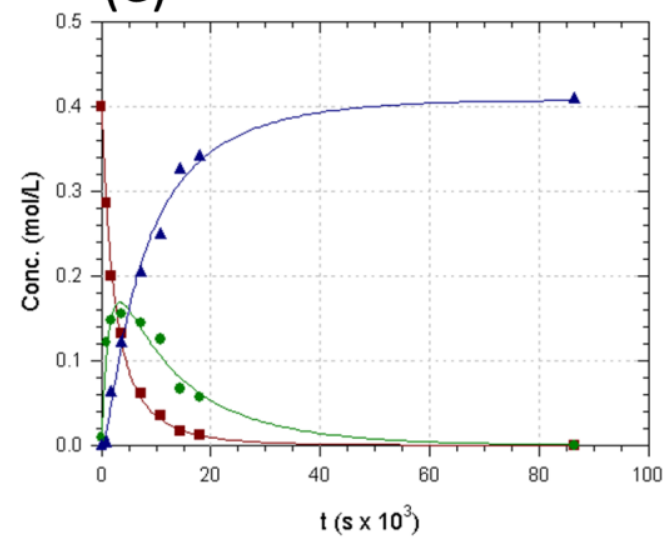

(b)

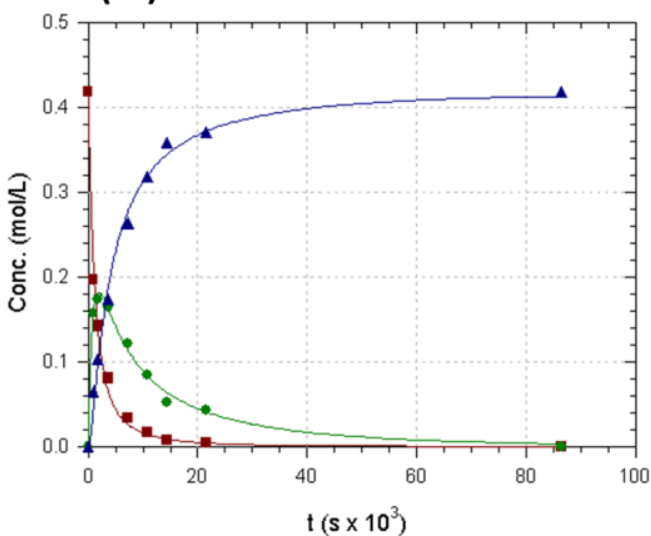

(d)

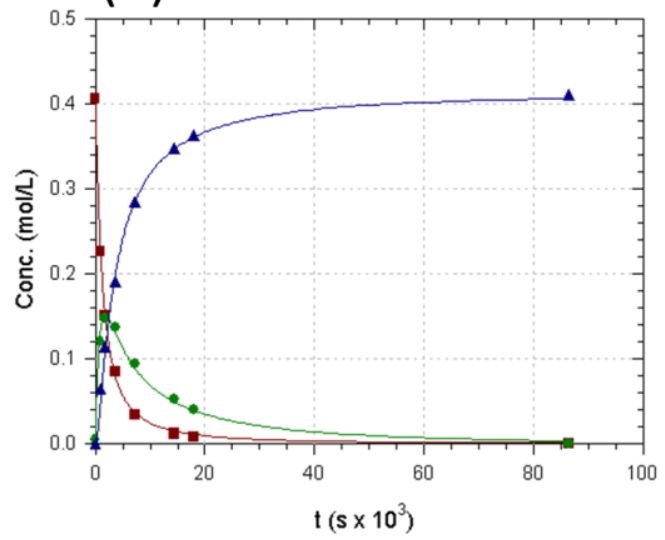

Figure S9. Kinetic traces for the oxidation of methyl $p$-tolyl sulfide (red squares) to its sulfoxide (green circles) and sulfone (blue triangles) by hydrogen peroxide in the presence of (a) S10, (b) L7, (c) S7, (d) $\mathbf{L 5}$, (e) $\mathbf{S 5}$ as heterogeneous catalyst at $50{ }^{\circ} \mathrm{C}$. The $\mathbf{Z r}_{\mathbf{4}}$ is present in $0.1 \mathrm{~mol} \%$ respect to the methyl $p$-tolyl sulfide. Symbols: experimental points; lines: second order kinetic fitting. 

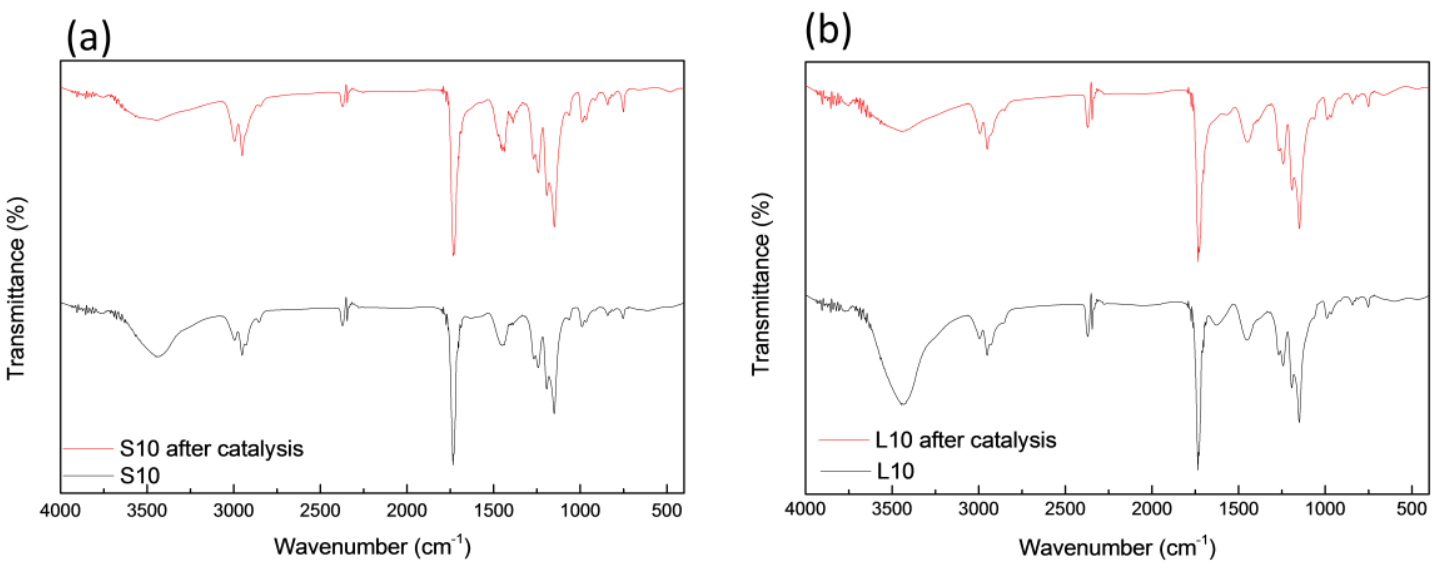

Figure S10. FTIR spectra of (a) S10 before and after catalysis and (b) L10 before and after catalysis.

Hybrid poly(MMA-co-Zrr $\left.\mathbf{Z}_{4}\right)$ nanoparticles $\left(\mathrm{KBr}, \mathrm{cm}^{-1}\right)$ : 3449 (broad); 2993 (medium, symmetric CH); 2952 (medium, CH); 2929 (medium, CH); 2853 (weak, CH); 1735 (strong, symmetric COOMe); 1630 (weak, $\mathrm{C}=\mathrm{C}$ ); 1453 (medium, bending $\mathrm{CH}_{3}$ ); 1270 (medium); 1244 (medium); 1194 (medium); 1148 (medium, bending CO); 1063 (medium); 990 (medium); 966 (medium); 840 (weak); 809 (medium); 752 (medium).

PMMA nanoparticles ( $\mathrm{KBr}, \mathrm{cm}^{-1}$ ): 3449 (broad); 2993 (medium, symmetric $\mathrm{CH}$ ); 2952 (medium, CH); 2929 (medium, CH); 2853 (weak, CH); 1735 (strong, symmetric COOMe); 1630 (weak, C=C); 1453 (medium, bending $\mathrm{CH}_{3}$ ); 1270 (medium); 1244 (medium); 1194 (medium); 1148 (medium, bending CO); 1063 (medium); 990 (medium); 966 (medium); 840 (weak); 809 (medium); 752 (medium). 
(a)

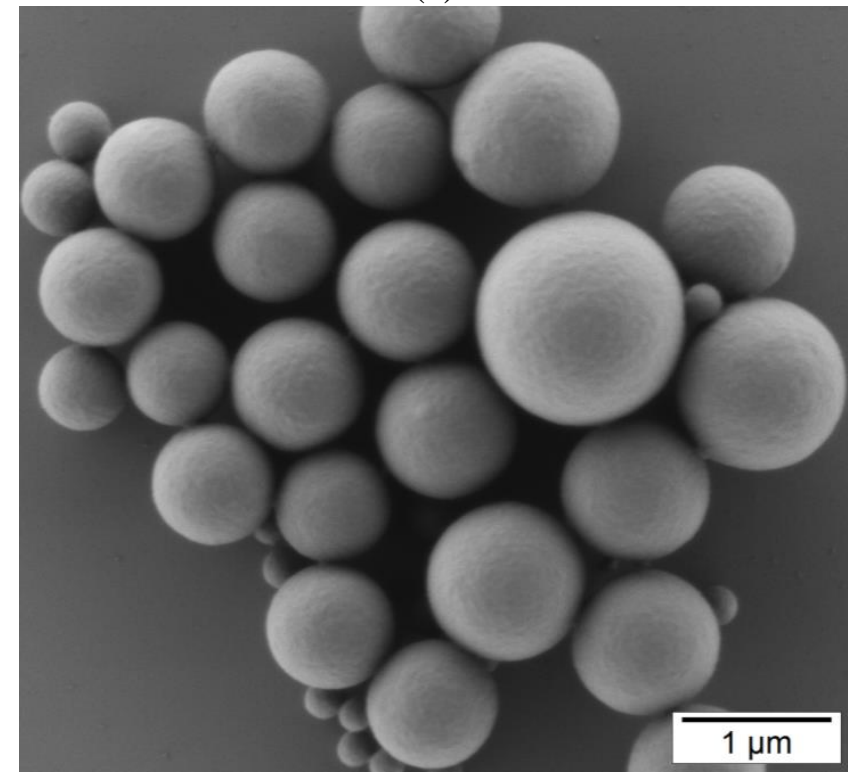

(b)

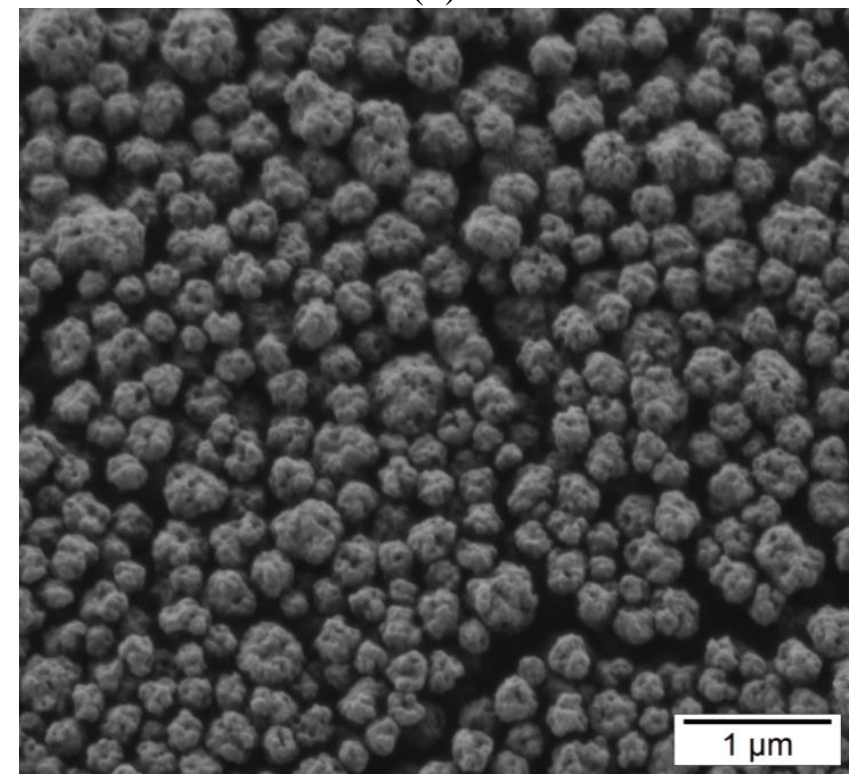

Figure S11. SEM images of the sample (a) S10 and (b) L10 after catalysis. The picture on the left clearly show the consequence of the lower cross-linking efficacy of $\mathbf{L 1 0}$.

\subsection{ICP after catalysis}

After the initial washing step, the catalysis and recover of the catalyst by centrifugation the non-cross-linked part of the nanoparticles matrix is not present anymore due to the solubility of the linear polymer. Therefore, the ICP measurements done are referred to the $\mathbf{Z r}_{\mathbf{4}}$ content in only the cross-linked part. The $\mathbf{Z r}_{4}$ contents after the catalysis are in agreement to calculated ones.

Table S3. Theoretical and determined $\mathbf{Z r}_{4}$ content of the particles after catalysis

\begin{tabular}{ccc}
\hline Sample & $\begin{array}{c}\text { Calculated } \mathbf{Z r}_{\mathbf{4}} \text { content in the cross- } \\
\text { linked part }(\mathrm{wt} \%)\end{array}$ & $\begin{array}{c}\mathbf{Z} \mathbf{r}_{\mathbf{4}} \text { content by ICP after the catalysis } \\
(\mathrm{wt} \%)^{\mathrm{a}}\end{array}$ \\
\hline S10 & $10.9 \pm 0.1$ & $10.5 \pm 0.5$ \\
$\mathbf{L 1 0}$ & $13.1 \pm 0.1$ & $14.7 \pm 0.5$ \\
\hline
\end{tabular}

\footnotetext{
${ }^{\text {[a] }}$ Determined by ICP-OES.
} 
(a)

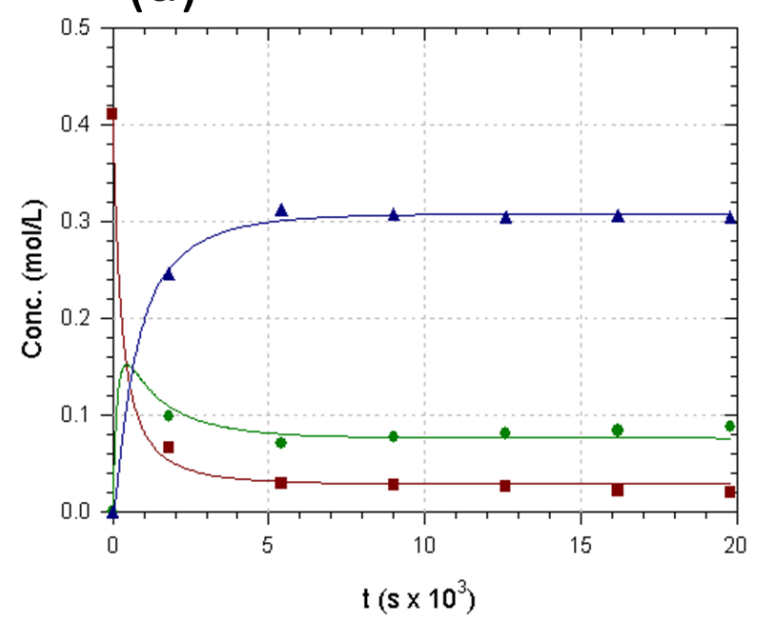

(b)

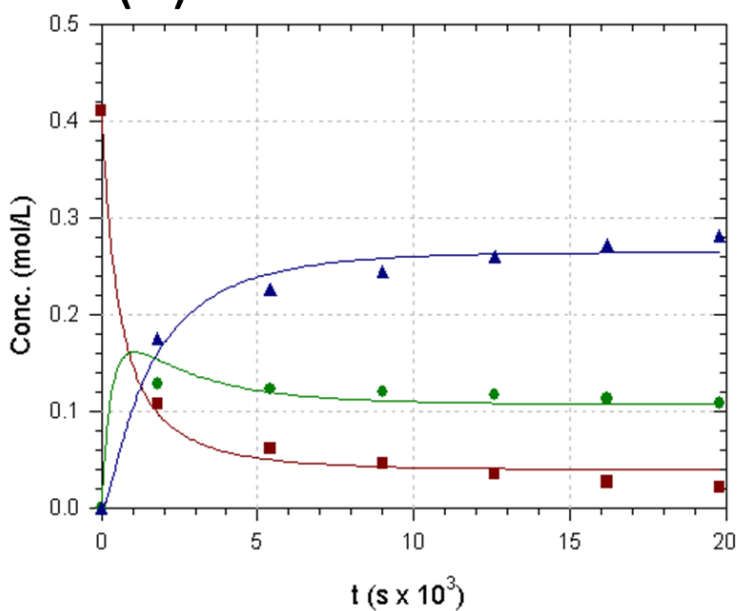

Figure S12. Kinetic traces for the oxidation of methyl $p$-tolyl sulfide (red squares) to its sulfoxide (green circles) and sulfone (blue triangles) by hydrogen peroxide in the presence of (a) $\mathbf{L 1 0}$ and (b) $\mathbf{L} 7$ as heterogeneous catalyst at $50{ }^{\circ} \mathrm{C}$. The $\mathbf{Z r}_{\mathbf{4}}$ is present in $0.1 \mathrm{~mol} \%$ respect to the methyl $p$-tolyl sulfide. The catalysts were removed after $30 \mathrm{~min}$ by high speed centrifugation.

(a)

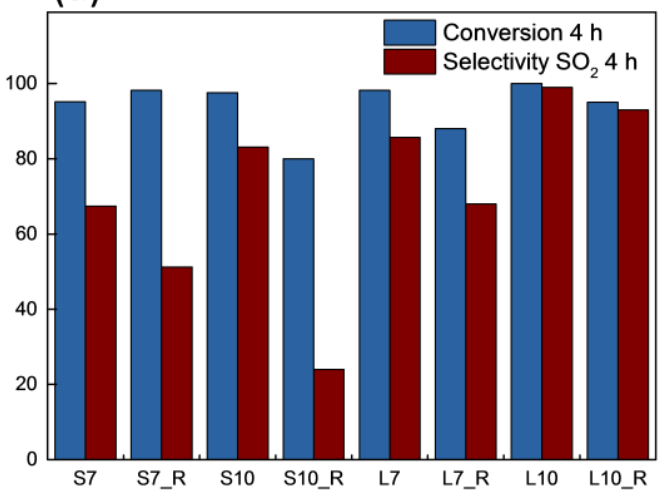

(b)

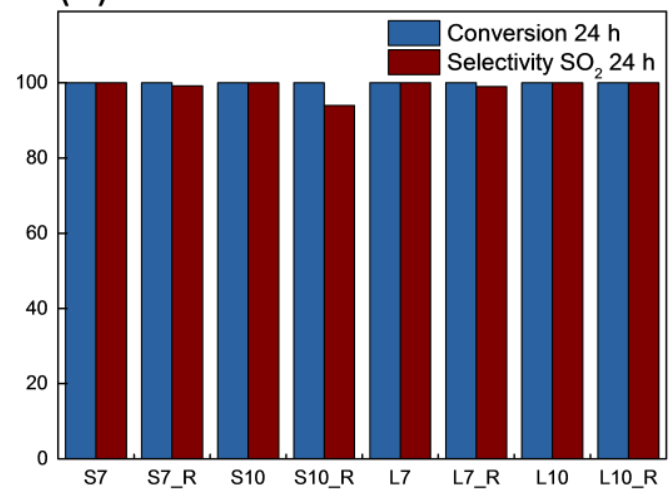

Figure S13. The methyl $p$-tolyl sulfide conversion and the selectivity to the sulfone at (a) $4 \mathrm{~h}$ and (b) $24 \mathrm{~h}$ in the presence of S7, S10, L7 and L10 in the first cycle and after recycling of the catalysts with a fresh mixture of sulfide and $\mathrm{H}_{2} \mathrm{O}_{2}$ (labeled as “_R”). 


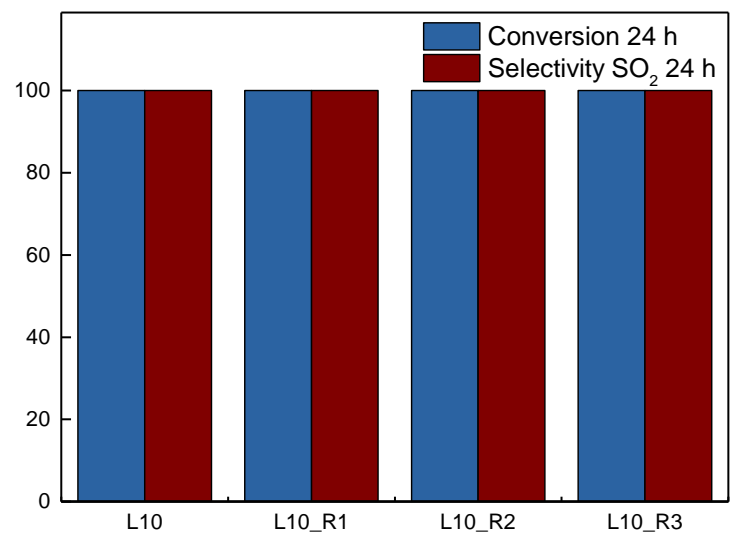

Figure S14. The methyl $p$-tolyl sulfide conversion and the selectivity towards the sulfone at $24 \mathrm{~h}$ in the presence of $\mathbf{L 1 0}$ in the first cycle and after three recycles of the catalysts with a fresh mixture of sulfide and $\mathrm{H}_{2} \mathrm{O}_{2}$ (labeled as “_R1”, “_R2”, “_R3” for the first, second and third recycle, respectively). 